\title{
Synchronous and Asynchronous Temperature Modulation of Rayleigh- Bénard Chandrasekhar Convection in Couple Stress Fluid using Ginzburg-Landau Model
}

\author{
Neha Aanam A ${ }^{1}$ and S. Pranesh ${ }^{1}$ \\ ${ }^{1}$ Department of Mathematics, CHRIST (Deemed to be University), Bengaluru 29, India.
}

\begin{abstract}
We consider a non-linear theory to investigate the transfer of heat in a layer of a couple stress fluid with temperature modulation. The influence of synchronous and asynchronous temperature modulation on the onset of Rayleigh-Bénard Chandrasekhar convection has been investigated. A nonautonomous Ginzburg-Landau equation for the amplitude of convection is obtained and Nusselt number expression is arrived at analytically by expanding the disturbances as a power series of amplitude of modulation. We give a detailed report of the effect of heat transfer by quantifying in terms of the Nusselt number for various system parameters. The Chandrasekhar number, Q stabilizes the system by decreasing the heat transfer while the system is destabilized for the couple stress parameter in both the modulations, resulting in an increased heat transfer across the fluid. Furthermore, it is found that an effective use of temperature modulation can aid in regulating the heat transfer.
\end{abstract}

Keywords: Non-Linear Analysis, Temperature Modulation, Couple Stress fluid, Ginzburg-Landau Equation, Heat Transfer

\section{INTRODUCTION}

The predominant motive for the study of the Rayleigh-Be'nard Chandrasekhar convection is due to its innumerable applications in the fields of geophysics, astrophysics and in specific the study of sunspots. The presence of strong magnetic fields in the earth's elemental metallic layers and interiors of stellar find fundamental applications in the study of convection. Rayleigh-Bénard Chandrasekhar convection exhibits various behaviors with the governing system leading to both stable (steady) and unstable (oscillatory) solutions when the inverse ratio of the thermal diffusivity to the magnetic field is small. Magnetic convection helps in controlling the stability and heat transfer in the system. A non-dimensional number called as Chandrasekhar number (Q) measures the strength of the magnetic field which is given as the ratio of the Lorentz force to the viscosity. It is found that the onset of convection is inhibited if the Lorentz force is greater than the viscous force. Furthermore it is discerned that the application of magnetic field twists and stretches the motion of the fluid for Lorentz force less than the viscous force. Furthermore increasing magnetic field gives rise to the turbulence in the fluid [27]. The flow behavior of various hydrodynamic systems changes in response to parametric modulation of driving force. Also the heating of a fluid from below generally gives rise to thermal convective instability. Rayleigh-Bénard convection [1, 2, 3, $4]$ is one of the most interesting and well-studied problem of instability phenomena. The maintenance of space dependent erratic temperature gradient is one of the potent mechanisms of controlling convection ([5], [6] and [7]). Nevertheless, transitory heating or cooling at the boundaries gives rise to nonuniform temperature gradients in various pragmatic situations. Hence this basic temperature profile is explicitly dependent on position and time. This problem which presumes the energy equation as a solution under appropriate boundary conditions which are time-dependent is referred to as the thermal modulation problem. Furthermore, an appropriate tuning of the parameters like, amplitude and frequency of modulation can aid in controlling the convective flow as these temperature profiles can serve as an effective mechanism. Henceforth with an effective use of an imposed thermal modulation it is possible to enhance heat transfer in-order to destabilize an otherwise stable system. As this aids in attaining higher efficiencies in the applications of material processing. Likewise, in various processing techniques like solidification process it is possible to obtain higher efficiency by delaying the heat transfer inorder to stabilize an otherwise unstable system. For instance, time-dependent temperature gradients can be considered during solidification of metallic alloys in crystal growth to influence the heat transfer, as this aids in controlling the structure and quality of the resulting solid.

The investigation of the effect of time-sensitive heating in a horizontal fluid layer by Venezian [13] on the outset of thermal convection is a pathfinder work in this field. It is with the motivation by the experiment of Donnelly [12] that Venezian came up with this theory. The work by Donnelly involved the investigation of the effect of modulation due to rotational speed on the outset of instability between two concentric cylinders in a fluid flow. Varied researchers have presented an outstanding review of the diverse temperature modulation problems and their applications related to classical Rayleigh-Bénard convection in books of Ingham and Pop [8], Nield and Bejan [5] and Vafai [9]. Many authors (Bhaduria [10], Siddheshwar et. al. [6], Pranesh and Sangeetha [11]) have studied the influence of modulation on the onset of convection due to temperature under varying conditions. All the above literature is with respect to linear case.

Depending on various control parameters the innumerable problems of physical importance can be modeled in terms of a nonlinear partial differential equation. Also, the study of 
stability of fluids pertinent to divergent physical configurations arises a quest in the researchers for an exhaustive comprehension. It is a foremost priority to understand the stability of the fluid in these configurations in-order to know under what circumstances this fluid is stable or unstable. However, the stability of a particular flow can be determined by performing a stability analysis in the linear case. But, the fortuity of the growing disturbance just above the threshold cannot be predicted using this linear theory. Thus manifestations based on the experimental evidence indicate that the basic state loses its stability with an increase in the control parameter above some critical value and in the perturbed state a pattern with a more complicated structure evolves and this can be periodic or quasi-periodic or chaotic in nature.

The broad spectrum of applications of buoyancy driven convection of non-Newtonian fluids like couple stress fluids provides the framework and incentive for the problem to be studied by this paper. There lies a huge necessity to understand the complexity of the couple stress fluid theory as this emerging area of application of couple stress fluids paves way for advanced research accompanied by broad range of ideas, challenges and applications. Couple stress is the culmination of an assumption that the force and moment distribution acting on the body across a surface are equivalent to the mechanical action of one part of a body on another. Siddheshwar and Pranesh [14] gave a detailed description in their introductory work in couple stress fluids for the Rayleigh-Bénard problem. The study included an analysis of both linear and non-linear stability analyses for the Rayleigh-Bénard convection in a couple-stress fluid layer. In the recent past, pertaining to the significance of couple stress fluids in various applications of chemical engineering, viz., liquid crystals, lubrication of engine rod bearings [15], polymer-thickened oils, tribology of thrust bearings [16], polymeric suspensions [17] and physiological fluid mechanics [18] researchers are involved in the comprehensive study of couple stress fluids.

Non-linear convection which has been a vital phenomenon in the study of practically important fluids needs a thorough comprehension to broaden this area of research and hence it becomes a huge necessity to determine the amount of heat transfer, finite amplitude, and various distinct characteristics. Lack of periodicity is inherently a familiar characteristic of various phenomena of nature. The Ginzburg-Landau Equation (GLE) can be considered as a generic model equation, describing the nonlinear evolution of patterns which evolve in the case where the basic state of a given physical system becomes unstable. Varied researchers have derived the GLE independently. A GLE with real coefficients for the case of Bénard convection was derived by Newell and Whitehead [19]. In 1965, L.D. Landau and V.L. Ginzburg posed the stationary equivalent of the equation in a paper on superconductivity [20]. Depending on the values of the Ginzburg-Landau constants the GLE is used to predominantly model the spatio-temporal dynamics of complex flows as they present a substantial variety of solutions. Undoubtedly the Rayleigh- Bénard Chandrasekhar convection problem has been substantially explored for Newtonian fluids; however the problems of non-Newtonian fluids have got a lesser recognition. With this motivation accompanied by the enormous applications of couple stress fluids, the prime objective of this paper is to study the effect of temperature modulation on heat transfer in Rayleigh- Bénard Chandrasekhar convection in couple stress fluids for synchronous and asynchronous temperature modulations using Ginzburg-Landau equation.

\section{MATHEMATICAL FORMULATION}

The physical configuration of the problem consists of a couple stress fluid taken between two horizontal surfaces of infinite length separated by a distance'd'. Let $\Delta T$ be the temperature difference between the lower and upper plate. We consider a coordinate system in a Cartesian plane with the lower boundary in its origin and z-axis vertically upwards. We assume that the boundaries are vitally free in addition to being ideal heat conductors in the absence of tension on the surface of the fluid. An externally applied uniform magnetic field $H_{0}$ is applied normal to the fluid layer as shown in Figure 1.

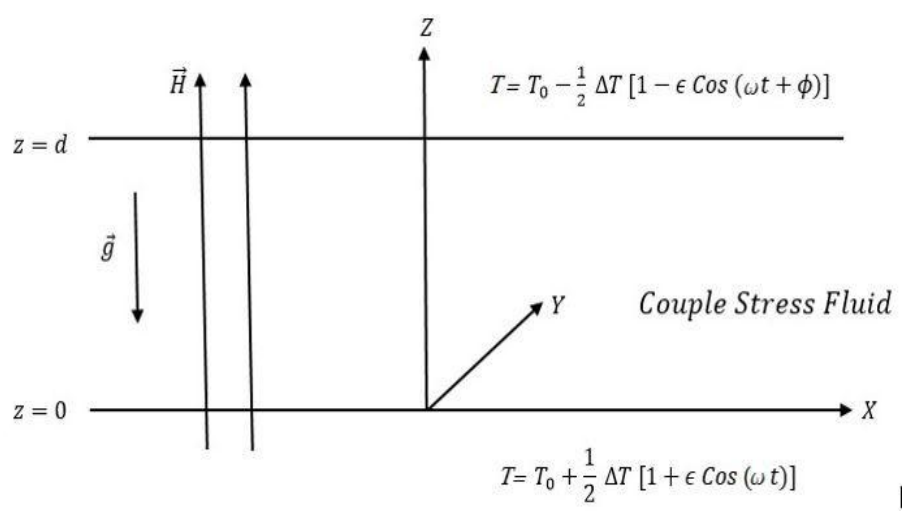

Figure 1: Physical configuration of the problem.

Under the Boussinesq approximation, the equations which govern this incompressible couple stress fluid which is electrically conducting are given by

$\nabla \cdot \vec{q}=0$,

$\rho_{0}\left[\frac{\partial \vec{q}}{\partial t}+(\vec{q} \cdot \nabla) \vec{q}\right]=-\nabla P+\rho g_{0} \hat{k}+\mu \nabla^{2} \vec{q}-\mu^{\prime} \nabla^{4} \vec{q}$ $+\mu_{m}(\vec{H} \cdot \nabla) \vec{H}$

$\frac{\partial T}{\partial t}+(\vec{q} \cdot \nabla) T=\chi \nabla^{2} T$

$\rho=\rho_{0}\left[1-\alpha\left(T-T_{0}\right)\right]$,

$\frac{\partial \vec{H}}{\partial t}+(\vec{q} \cdot \nabla) \vec{H}=\gamma_{m} \nabla^{2} \vec{H}+(\vec{H} \cdot \nabla) \vec{q}$,

$\nabla \cdot \vec{H}=0$. 
To study the problem we use the time-dependent boundary conditions at the lower and upper boundaries given by Venezian [13]

$$
\left.\begin{array}{r}
T(0, t)=T_{0}+\frac{1}{2} \Delta T\left[1+\epsilon^{2} \delta \operatorname{Cos} \omega t\right], \\
T(d, t)=T_{0}-\frac{1}{2} \Delta T\left[1+\epsilon^{2} \delta \operatorname{Cos}(\omega t+\phi)\right] .
\end{array}\right\}
$$

The fluid is considered to be at rest and is given by

$\vec{q}=\vec{q}_{b}=0, \rho=\rho_{b}(z, t), T=T_{b}(z, t), P=P_{b}(z, t), \vec{H}_{b}=H_{0} \hat{k}$.

The basic state quantities viz., temperature $T_{b}$, pressure $P_{b}$ and density $\rho_{b}$ are governed by the following equations:

$\frac{\partial T_{b}}{\partial t}=\chi \frac{\partial^{2} T_{b}}{\partial z^{2}}$

$\frac{\partial P_{b}}{\partial z}=-\rho_{b} g \hat{k}+\mu_{m}(\vec{H} \cdot \nabla) \vec{H}$

$\rho_{b}=\rho_{0}\left(1-\alpha\left(T_{b}-T_{0}\right)\right)$.

The solution of (9) subject to the boundary conditions (7) consists of a steady part $\left(T_{S}\right)$ and an unsteady oscillatory part $\left(\epsilon T_{1}\right)$ and is given by

$T_{b}(z, t)=T_{s}(z)+\epsilon^{2} \delta R e\left[T_{1}(z, t)\right]$.

$T_{s}(z)=T_{0}+\frac{\Delta T}{2 d}(d-2 z)$,

$T_{1}(z, t)=\operatorname{Re}\left\{\left[a(\lambda) e^{\frac{\lambda z}{d}}+a(-\lambda) e^{\frac{-\lambda z}{d}}\right] e^{-i \omega t}\right\}$

where,

$a(\lambda)=\frac{\Delta T}{2}\left[\frac{e^{-i \phi}-e^{-\lambda}}{e^{\lambda}-e^{-\lambda}}\right], \quad \lambda=(1-i) \sqrt{\frac{\omega}{2}}$

and $R e$ denotes the real part.

We now investigate the stability for the non-linear case by superposing infinitesimal disturbances on this basic state. Thus by assuming finite amplitude disturbances on the basic state we obtain the following perturbed quantities:

$\vec{q}=\vec{q}_{b}+\vec{q}^{\prime}, \quad P=P_{b}+P^{\prime}, \quad \rho=\rho_{b}+\rho^{\prime}$,

$T=T_{b}+T^{\prime}, \quad \vec{H}=\vec{H}_{b}+\vec{H}^{\prime}$

The prime stipulates that the quantities are infinitesimal perturbations. Substituting (16) into equations (1) to (6) and using the solutions (9) to (11) of the basic state, we get the following equations which govern the perturbations of the infinitesimal type.

$$
\begin{aligned}
\rho_{0}\left[\frac{\partial q}{\partial t}\right]+\left(\overrightarrow{q^{\prime}} \cdot \nabla\right) \cdot \overrightarrow{q^{\prime}} & =-\nabla P^{\prime}+\rho \vec{g}+\mu \nabla^{2} \overrightarrow{q^{\prime}} \\
& -\mu^{\prime} \nabla^{4} \overrightarrow{q^{\prime}}+\mu_{m} H_{0} \frac{\partial \overrightarrow{H^{\prime}}}{\partial z}
\end{aligned}
$$

$$
\begin{aligned}
& \nabla \cdot \vec{q}^{\prime}=0 \\
& \frac{\partial T^{\prime}}{\partial t}+\left(\overrightarrow{q^{\prime}} \cdot \nabla\right) \cdot \overrightarrow{T^{\prime}}+w^{\prime} \frac{\partial T_{b}}{\partial z}=\chi \nabla^{2} T, \\
& \frac{\partial \overrightarrow{H^{\prime}}}{\partial t}+\left(\overrightarrow{q^{\prime}} \cdot \nabla\right) \cdot \vec{H}^{\prime}=H_{0} \frac{\partial w^{\prime}}{\partial z} \hat{k}+\gamma_{m} \nabla^{2} \vec{H}^{\prime} \\
& \rho^{\prime}=-\alpha \rho_{0} T^{\prime}, \\
& \nabla \cdot \overrightarrow{H^{\prime}}=0 .
\end{aligned}
$$

The perturbations under consideration in our study are typically of two dimensions. The stream functions for velocity $\psi$ and magnetic potential $\varphi_{H}$ are introduced in the form $u^{\prime}=\frac{\partial \psi}{\partial z}, w^{\prime}=-\frac{\partial \psi}{\partial x}, H_{x}^{\prime}=\frac{\partial \phi_{H}}{\partial z}, \vec{H}_{z}^{\prime}=-\frac{\partial \phi_{H}}{\partial x} \quad$ which satisfy the continuity equation (17) and the magnetic continuity equation (22).

We non-dimensionalize the perturbed equations (18), (19) and (20) using the following denotations

$$
\begin{gathered}
\left(x^{*}, y^{*}, z^{*}\right)=\left(\frac{x^{\prime}}{d}, \frac{y^{\prime}}{d}, \frac{z^{\prime}}{d}\right), \quad t^{*}=\frac{t^{\prime}}{d^{2} / \chi}, \quad \overrightarrow{q^{*}}=\frac{\overrightarrow{q^{\prime}}}{\chi / d} \\
T^{*}=\frac{T^{\prime}}{\Delta T}, \quad \overrightarrow{H^{*}}=\frac{\vec{H}^{\prime}}{H_{0}}, \quad P^{*}=\frac{P^{\prime}}{P_{0}} \\
\psi^{*}=\frac{\psi}{\chi}, \quad w^{*}=\frac{w^{\prime}}{\chi / d^{2}} .
\end{gathered}
$$

Using equation (21) in (18), introducing the stream functions $\psi$ and $\varphi_{H}$, cross differentiating to eliminate the pressure term and non-dimensionalizing the resultant equations using equation (23) we get the following dimensionless equations:

$$
\begin{aligned}
& \frac{1}{\operatorname{Pr}}\left[\frac{\partial}{\partial t}\left(\nabla^{2} \psi\right)-J\left(\psi, \nabla^{2} \psi\right)\right]-\nabla^{4} \psi+C \nabla^{6} \psi= \\
& -R \frac{\partial T}{\partial x}+Q P_{m} \frac{\partial}{\partial z}\left(\nabla^{2} \phi\right)-Q P_{m} J\left(\phi, \nabla^{2} \phi\right), \\
& \frac{\partial \psi}{\partial x} \frac{\partial T_{0}}{\partial z}-\nabla^{2} T=-\frac{\partial T}{\partial t}+\epsilon^{2} \delta f_{2}(z, t) \frac{\partial \psi}{\partial x}+J(\psi, T) \\
& -\frac{\partial \psi}{\partial z}-P_{m} \nabla^{2} \phi=-\frac{\partial \phi}{\partial t}+J(\psi, \phi) .
\end{aligned}
$$

The dimensionless parameters in the above equations are

Rayleigh Number, $\quad R=\frac{\alpha g \Delta T d^{3} \rho_{0}}{\mu \chi}$;

Chandrasekhar Number, $Q=\frac{\mu_{m}^{2} H^{2} d^{2} \sigma}{\mu}$

Prandtl Number, $\quad \operatorname{Pr}=\frac{\mu}{\rho_{0} \chi}$;

Magnetic Prandtl Number, $P m=\frac{\mu}{\rho_{0} \gamma_{m}}$

Couple Stress Parameter, $C=\frac{\mu^{\prime}}{\mu d^{2}} \quad\left(0 \leq C \leq m_{)}\right.$,

where $m$ is a finite, positive real number according to the Clasusius - Duhem inequality.

In (25) $\frac{\partial T_{0}}{\partial z}$ is the non-dimensional form of $\frac{\partial T_{b}}{\partial z}$ and is given by: 
$\frac{\partial T_{0}}{\partial z}=-1+\epsilon^{2} \delta f_{2}(z, t)$

where,

$f_{2}(z, t)=\operatorname{Re}\left[f(z) e^{-i \omega}\right]$

$f(z)=\left[A(\lambda) e^{\lambda z}+\left[A(-\lambda) e^{-\lambda z}\right]\right.$,

We consider the following stress free boundary conditions which are maintained at constant temperature (isothermal) to solve the above system (30).

$\psi=0=\nabla^{2} \psi, \varphi_{H}=D \varphi_{H}=0$ at $z=0$,

$\psi=0=\nabla^{2} \psi, D \varphi_{H}=0 \quad$ at $z=1$.

where, $D=\partial / \partial z$

We use the assumptions of Govender [21] and Siddheshwar et al. $[22,23]$ to re-scale the time $\mathrm{t}$ as $\tau=\epsilon^{2} t$ in-order to maintain a slow time variation. This assumption thus aids to have small amplitude of modulations. We now proceed to study the convection of the system for the stationary case, and hence the nonlinear equations (24) to (26) are written as follows in the matrix form:

$\left[\begin{array}{ccc}-\nabla^{4}+C \nabla^{6} & R_{0 c} \frac{\partial}{\partial x} & -Q P_{m} \frac{\partial}{\partial z} \nabla^{2} \\ \frac{\partial}{\partial x} & -\nabla^{2} & 0 \\ \frac{\partial}{\partial z} & 0 & -P_{m} \nabla^{2}\end{array}\right]\left[\begin{array}{l}\psi \\ T \\ \phi_{H}\end{array}\right]$

$=\left[\begin{array}{c}-\frac{1}{\operatorname{Pr}}\left[\frac{\partial}{\partial \tau}\left(\nabla^{2} \psi\right)+\frac{1}{\operatorname{Pr}} J\left(\psi, \nabla^{2} \psi\right)\right]-Q P_{m} J\left(\phi_{H}, \nabla^{2} \phi_{H}\right) \\ -\frac{\partial T}{\partial \tau}+\epsilon^{2} \delta f_{2}(z, t) \frac{\partial \psi}{\partial x}+J(\psi, T) \\ -\frac{\partial \phi_{H}}{\partial \tau}+J\left(\psi, \phi_{H}\right) .\end{array}\right]$

\section{FINITE AMPLITUDE EQUATION}

We now introduce the subsequent asymptotic expansions (these expansions are in accordance with those suggested by Malkus et al. [24] and Venezian [13]) to resolve the nonlinearity:

$$
\begin{array}{r}
R=R_{0 c}+\epsilon^{2} R_{2 c}+\epsilon^{4} R_{4}+\ldots \\
\psi=\epsilon \psi_{1}(x, z, t)+\epsilon^{2} \psi_{2}(x, z, t)+\epsilon^{3} \psi_{3}(x, z, t)+\ldots \\
T=\epsilon T_{1}(x, z, t)+\epsilon^{2} T_{2}(x, z, t)+\epsilon^{3} T_{3}(x, z, t)+\ldots \\
\phi_{H}=\epsilon \phi_{H 1}(x, z, t)+\epsilon^{2} \phi_{H 2}(x, z, t)+\epsilon^{3} \phi_{H 3}(x, z, t)+\ldots
\end{array}
$$

where $R_{0 c}$ is the critical value of the Rayleigh number. In the absence of temperature modulation, the onset of the RayleighBénard Chandrasekhar convection occurs at this critical value of the Rayleigh number. The system (30) is now solved for different orders of $\epsilon$.

At the lowest order the above system (30) takes the following form:

$$
\left[\begin{array}{ccc}
-\nabla^{4}+C \nabla^{6} & R_{0 c} \frac{\partial}{\partial x} & -Q P_{m} \frac{\partial}{\partial z} \nabla^{2} \\
\frac{\partial}{\partial x} & -\nabla^{2} & 0 \\
\frac{\partial}{\partial z} & 0 & -P_{m} \nabla^{2}
\end{array}\right]\left[\begin{array}{c}
\psi_{1} \\
T_{1} \\
\phi_{H 1}
\end{array}\right]=\left[\begin{array}{l}
0 \\
0 \\
0
\end{array}\right]
$$

This order defines the Rayleigh number at its critical point given by $R_{0 c}$ in equation (35). Also the critical wave number obtained is similar to that of linear stability analysis [11]. Under the boundary conditions (31), solutions of the system at its lowest order is assumed to be

$$
\begin{array}{r}
\psi_{1}=A(\tau) \operatorname{Sin}\left(k_{c} x\right) \operatorname{Sin}(\pi z) \\
T_{1}=\frac{k_{c}}{\delta^{2}} A(\tau) \operatorname{Cos}\left(k_{c} x\right) \operatorname{Sin}(\pi z), \\
\phi_{H 1}=\frac{\pi}{P_{m} \delta^{2}} A(\tau) \operatorname{Sin}\left(k_{c} x\right) \operatorname{Cos}(\pi z) .
\end{array}
$$

We arrive at the expression for the Rayleigh number at its critical point for the onset of the Rayleigh- Bénard Chandrasekhar convection in the absence of temperature modulation as given by

$$
R_{0 c}=\frac{\delta^{6}}{k_{c}^{2}}+C \frac{\delta^{8}}{k_{c}^{2}}+Q \frac{\pi^{2} \delta^{2}}{k_{c}^{2}}
$$

Following limiting cases can be obtained from equation (35)

1. If $C=0, Q=0$, equation (35) reduces to the classical result forNewtonian fluid.

2. If $C \neq 0, Q=0$, equation (35) reduces to the expression obtained by Siddheshwar et al. [22] which is the same as the results obtained by Chandrasekhar [2] and Bhadauria et al. [10] for

$(R=0)$.

3. If $C=0, Q \neq 0$, equation (35) reduces to the Rayleigh number obtained by Chandrasekhar [2]

4. At the second order, we have

$$
\left[\begin{array}{ccc}
-\nabla^{4}+C \nabla^{6} & R_{0 c} \frac{\partial}{\partial x} & -Q P_{m} \frac{\partial}{\partial z} \nabla^{2} \\
\frac{\partial}{\partial x} & -\nabla^{2} & 0 \\
\frac{\partial}{\partial z} & 0 & -P_{m} \nabla^{2}
\end{array}\right]\left[\begin{array}{c}
\psi_{2} \\
T_{2} \\
\phi_{H 2}
\end{array}\right]=\left[\begin{array}{c}
R_{21} \\
R_{22} \\
R_{23} .
\end{array}\right]
$$

where,

$R_{21}=Q P_{m} \frac{\partial}{\partial z}\left(\nabla^{2} \phi\right)-Q P_{m} J\left(\phi, \nabla^{2} \phi\right)=0$

$R_{22}=\frac{-A^{2}(\tau) k_{c}^{2} \pi}{2 \delta^{2}} \operatorname{Sin}(2 \pi z)$

$R_{23}=\frac{-A^{2}(\tau) k_{c} \pi^{2}}{2 P m \delta^{2}} \operatorname{Sin}\left(2 k_{c} x\right)$

The solutions of the second order system (36) subjected to the boundary conditions (31) are obtained as follows:

$\psi_{2}=0$,

$$
\begin{aligned}
& T_{2}=\frac{k_{c}^{2}}{8 \pi \delta^{2}} A^{2}(\tau) \operatorname{Sin}(2 \pi z) \\
& \phi_{H 2}=\frac{-\pi^{2}}{8 k_{c}^{2} P_{m} \delta^{2}} A^{2}(\tau) \operatorname{Sin}\left(2 k_{c} x\right)
\end{aligned}
$$


We quantify this amount of heat transfer in terms of the horizontally averaged Nusselt number, $N u(\tau)$ and is defined as follows:

$$
N u(\tau)=1+\frac{\left[\frac{k_{c}}{2 \pi} \int_{0}^{\frac{2 \pi}{k_{c}}}\left(\frac{\partial T_{2}}{\partial z}\right) d x\right]_{z=0}}{\left[\frac{k_{c}}{2 \pi} \int_{0}^{\frac{2 \pi}{k_{c}}}\left(\frac{\partial T_{b}}{\partial z}\right) d x\right]_{z=0}}
$$

where the subscript in the integrand denotes the derivative with respect to $\mathrm{Z}$. Also, it becomes necessary to choose an appropriate time interval for a thorough comprehension of the effect of temperature modulation on heat transport. Thus we discern from the studies of Siddheshwar et al. [25] that the interval $[0,2 \pi]$ is suitable to calculate the mean Nusselt number. Thus on substituting for $T_{2}$ and $T_{b}$ in (38) and simplifying we obtain the following expression for the Nusselt number:

$$
N u(\tau)=1+\frac{k_{c}^{2}}{4 \delta^{2}} A^{2}(\tau)
$$

At the third order, we have

$$
\begin{aligned}
& {\left[\begin{array}{ccc}
-\nabla^{4}+C \nabla^{6} & R_{0 c} \frac{\partial}{\partial x} & -Q P_{m} \frac{\partial}{\partial z} \nabla^{2} \\
\frac{\partial}{\partial x} & -\nabla^{2} & 0 \\
\frac{\partial}{\partial z} & 0 & -P_{m} \nabla^{2}
\end{array}\right]\left[\begin{array}{l}
\psi_{3} \\
T_{3} \\
\phi_{H 3}
\end{array}\right]=\left[\begin{array}{l}
R_{31} \\
R_{32} \\
R_{33}
\end{array}\right]} \\
& R_{31}=-\frac{1}{\operatorname{Pr}} \frac{\partial}{\partial t} \nabla^{2} \psi_{1}-R_{2 c} \frac{\partial T_{1}}{\partial x}-Q P_{m}\left[\frac{\partial \phi_{H 1}}{\partial z} \frac{\partial}{\partial x} \nabla^{2} \phi_{H 2}\right. \\
& \left.-\frac{\partial \phi_{H 2}}{\partial x} \frac{\partial}{\partial z} \nabla^{2} \phi_{H 1}\right] \\
& =\left[\frac{\epsilon^{2} \delta^{2}}{\operatorname{Pr}} A^{\prime}(\tau)-\frac{R_{2} k_{c}^{2}}{\delta^{2}} A(\tau)-Q P m L \operatorname{Cos}\left(2 k_{c} x\right) A^{2}(\tau)\right] \\
& \operatorname{Sin}\left(k_{c} x\right) \operatorname{Sin}(\pi z) \\
& R_{32}=-\frac{\partial T_{1}}{\partial \tau}+\delta f_{2}(z, t) \frac{\partial \psi_{1}}{\partial x}+\frac{\partial \psi_{1}}{\partial x} \frac{\partial T_{2}}{\partial z}, \\
& =\left\{k_{c} \epsilon \delta_{1} f_{2}(z, t) A(\tau)+\frac{k_{c}}{\delta^{2}}\left[A^{\prime}(\tau)-\frac{k_{c}^{2}}{4} A^{3}(\tau)+\right.\right. \\
& \left.\left.\frac{k_{c}^{2}}{2 \delta^{2}} \operatorname{Sin}^{2}(2 \pi z) A^{3}(\tau)\right]\right\} \operatorname{Cos}\left(k_{c} x\right) \operatorname{Sin}(\pi z)
\end{aligned}
$$

where $\delta f_{2}(z, t)=\frac{1.6 n^{2} \pi^{4} \omega^{2}}{\left[\omega^{2}+(n+1)^{4} \pi^{4}\right]\left[\omega^{2}+(n-1)^{4} \pi^{4}\right]}$

$$
\begin{aligned}
& R_{33}=-\frac{\partial \phi_{H 1}}{\partial \tau}-\frac{\partial \psi_{1}}{\partial z} \frac{\partial \phi_{H 2}}{\partial x} . \\
&=\left.\left.\left\{\frac{\pi}{P m \delta^{2}}\left[-A^{\prime}(\tau)+\frac{\pi^{2}}{4 P m} A^{3}(\tau)+\frac{\pi^{2}}{P m \delta^{2}}\right]\right)+\frac{\pi^{3}}{2 P m \delta^{2}}\right]\right\} \\
& \operatorname{Sin}\left(k_{c} x\right) \operatorname{Cos}(\pi z)
\end{aligned}
$$

The existence of the third order solution is validated by the solvability condition given by

$\int_{z=0}^{1} \int_{x=0}^{\frac{2 \pi}{k_{c}}}\left[\psi_{1} R_{31}+R_{0 c} T_{1} R_{32}+Q P_{m} \phi_{H 1} R_{33}\right] d x d z$.

By substituting for $R_{31}, R_{32}$ and $R_{33}$ in (44) we arrive at the nonautonomous

Ginzburg-Landau equation with time-periodic coefficients for the stationary mode of convection as given below,

$$
A_{1} A^{\prime}(\tau)-A_{2} A(\tau)+A_{3} A^{3}(\tau)=0,
$$

where,

$$
\begin{aligned}
& A_{1}=\frac{\delta^{2} \pi \epsilon^{2}}{2 P r k_{c}}-\frac{R_{0 c} \pi}{2 \delta^{4}}-\frac{Q \pi^{3}}{2 P_{m} \delta^{4} k_{c}}, \\
& A_{2}=\frac{R_{2 c} \pi k_{c}}{2 \delta^{2}}+\left(\frac{R_{0 c} \epsilon \pi k_{c} \delta_{1}}{\delta^{2}}\right) L(\tau) \\
& A_{3}=\frac{5 Q \pi^{5}}{16 P_{m}^{2} \delta^{4} k_{c}}+\frac{3 Q P_{m} \pi}{4 k_{c}}\left(-\frac{k_{c}^{2} \pi^{4}}{P_{m}^{3} \delta^{4}}+\frac{k_{c}^{2} \pi^{4}}{4 P_{m}^{3} \delta^{4}}+\frac{\pi^{6}}{4 P_{m}^{3} \delta^{4}}\right) \\
& L(\tau)=\int_{0}^{1} f_{2}(z, \tau) \operatorname{Sin}^{2} \pi z d z . \\
& 2 k_{c} \pi
\end{aligned}
$$

The non-autonomous nature of the Ginzburg-Landau equation (45) may pose its own difficulties to arrive at its analytical solution. To overcome this difficulty we use the inbuilt commands in Mathematica to obtain the solution by a numerical integration at time $\mathrm{t}=0$ (initial condition) with the initial amplitude of convection $A(0)=a_{0}$. Inorder to keep the parameters to a minimum, we assume that $R_{2 c}=R_{0 c}$ in the calculations.

\section{RESULTS AND DISCUSSIONS}

To investigate the instability in a fluid layer due to heating, the external regulation of convection becomes an important tool in a Rayleigh-Bénard convection problem. In this paper we have considered two such entities, viz., the vertical magnetic field and temperature modulation for either augmenting or impeding the amount of heat transfer due to convection as is statutory by the real life applications. This paper emphasizes the study of magneto-convection under temperature modulation in a couple stress fluid by using a non-autonomous Ginzburg-Landau equation. We study the effect of time-periodic modulated 
temperature on the onset of Rayleigh- Bénard Chandrasekhar convection in a fluid under the influence of couple stresses. It is imperative to consider a weak nonlinear theory to investigate and comprehend the nature and the amount of heat transfer as the linear theory does not complement this study. Furthermore, the modulated temperatures are taken to be of small orders as $O(\epsilon)^{2}$ which leads to small scales of amplitude of modulation. This kind of assumption aids in arriving at the equation of amplitude for convection in a rather unambiguous and sophisticated manner. Arriving at this equation of amplitude by the Ginzburg-Landau technique is much convenient than using the Lorenz model.

We discuss the effect of temperature modulation on the boundaries of the system in the following two cases:

\section{Synchronous Modulation $(\theta=0)$}

2. Asynchronous Modulation $(\theta=\pi)$

We study the effect of various parameters like Chandrasekhar number (Q), Couple stress parameter (C), Magnetic Prandtl number $(\mathrm{Pm})$ and the Prandtl number (Pr) on the onset of convection for different values of modulated frequencies $(\omega)$, amplitude of convection (A) and phase angle $(\varphi)$. The convective heat transfer in the system is influenced by the parameters Q, C, Pr, Pm which are associated with the fluid layer and the parameters $\varphi, A$ and $\omega$ are homologous to the external mechanisms of controlling convection. We discern the nature of heat transfer by keeping parameters constant one at a time. We assume low frequencies to modulate the temperatures at the boundaries as the effect of these lower range of frequencies is minimal on both the onset of convection and the convective heat transfer. We make the above assumption in order to ensure the inhibition of oscillations in the system at the onset of convection due to modulated temperatures. The influence of this modulated temperature is represented by the amplitude $\delta$, the property of the fluid is given by $\operatorname{Pr}$ while the effect of magnetic field is represented by $P m$ and $Q$. The Nusselt number quantifies the amount of heat transfer and its numerical values are obtained from (39) by elucidating the autonomous Ginzburg-Landau equation (45). The graphs of Nusselt number with respect to rescaled time $\tau$ are as seen in Figures (2a) - (5b). It is observed from the figures that temperature modulation either stabilizes or destabilizes the onset of convection as discussed below for different parameters. Also from the figures we observe that the Nusselt number value initiates at one and the system is in the conduction state with the value of this Nusselt number remaining a constant for some time. Later on the Nusselt number increases with time entering the convection mode. However, a further increase in time leads to a constant $\mathrm{Nu}$ thus reaching a steady state in general.

Figures (2a) and (4a) show the plot of time $\tau$ against the Nusselt number $N u$ for varying values of Chandrasekhar number $\mathrm{Q}$, for synchronous and asynchronous modulations respectively. The Chandrasekhar number delays the onset of convection and hence the heat transfer in the case of synchronous modulation as seen in Figure (2a). The effect of $N u$ begins with one; increases for small-scale values of time $\tau$ and turns out to be a constant for bigger values of time $\tau$. While in the case of asynchronous modulation as seen in Figure (4a) we observe an oscillatory behavior on the effect of $Q$. Furthermore an increase in $Q$ results in reduced transfer of heat quantified with the Nusselt number $(\mathrm{Nu})$ which implies that the system is stabilized due to an externally imposed magnetic field; these are the results [26] where the vertical magnetic field has stabilizing effect. Also, the magnetic lines are distorted by convection for stronger magnetic fields pervading the medium as this magnetic field influences the viscosity into the fluid. Furthermore the growth of disturbances in the system is hampered by these magnetic lines of force resulting in the impedance in the onset of instability in the system. However, the magnetic field applied on the system acts in the vertical direction thus allowing the Lorentz force to act horizontally. Hence the applied magnetic field will not allow free movement of the fluid in the vertical direction. This in-turn helps the fluid movement to be analogous to that in the case of absence of a magnetic field in the system. Hence the presence of magnetic field will bring in stability in the system. Increasing magnetic field will increase $\mathrm{Q}$, and the system will be more stabilizing. Hence $Q$ has a stabilizing result in synchronous and asynchronous modulations, resulting in reduces transfer of heat with $Q$.

Figures (2b) and (4b) manifests the influence of the couple stress parameter $\mathrm{C}$ for synchronous and asynchronous modulations respectively. In synchronous modulation we perceive from the figure (2b) that the influence of $N u$ on $\mathrm{C}$ starts with one; increases for minimum values of time $\tau$ and turns out to be a constant for bigger values of time $\tau$ while in the asynchronous modulation as in figure (4b) we observe an oscillatory behavior on the effect of $C$. Furthermore, an increase in $C$ results in an increased transfer of heat quantified with the Nusselt number $(\mathrm{Nu})$. This parameter $\mathrm{C}$ signifies the accumulation of the suspended particles and due to small modulation in the frequency, the influence of modulated temperatures is perceived at every point of the fluid layer and the system experiences an advanced onset of convection and thus augmenting the transfer of heat. Hence $C$ has a destabilizing effect in both the modulations, resulting in an increased heat transfer across the fluid.

Figures (3a) and (5a) represent the variations of the $\mathrm{Nu}$ profile against the slow time $\tau$ by keeping all the parameters constant except for varying values of magnetic Prandtl number (Pm). It is found that the magnetic Prandtl number influences an advancement in the onset of convection resulting in an increased transfer of heat. For the synchronous modulation the effect of $N u$ starts with one; increase for small-scale values of time $\tau$ and becomes a constant for large values of time $\tau$ as seen in figure (3a) while for asynchronous modulation, it is perceived that initially, $\mathrm{Nu}$ increases with increase in the magnetic Prandtl number but as the time passes the effect becomes negligible and the profile becomes oscillatory as seen in figure (5a). Hence $P m$ has a destabilizing effect in both the modulations, so that the amount of heat transfer increases with Pm.

Figures (3b) and (5b) represent the variations of the $\mathrm{Nu}$ profile with respect to slow time $\tau$ for varying values of Prandtl number (Pr) by keeping other parameters constant. It is found that the Prandtl number influences an advancement in the onset of 
convection and hence an increased transfer of heat. For the synchronous modulation the effect of $N u$ starts with one; increase for small values of time $\tau$ and turns out to be a constant for large values of time $\tau$ as seen in figure (3b) while for asynchronous modulation, it is observed that initially, $\mathrm{Nu}$ profiles increases with increase in Prandtl number but as the time passes the effect becomes negligible and the profile becomes oscillatory as seen in figure (5b).

The results of this study can be summarized as follows:

1. $[N u]_{Q=25}<[N u]_{Q=10}<[N u]_{Q=5}$, Figures (2a) and (4a)

2. $[N u]_{C}=0.15<[N u]_{C}=0.35<[N u]_{C}=0.55$, Figures $(2 \mathrm{~b})$ and (4b)

3. $[N u]_{P m}=0.75<[N u]_{P m}=0.77<[N u]_{P m}=0.79$, Figures (3a) and (5a)

4. $[N u]_{P r=4.5}<[N u]_{P r=5}<[N u]_{P m=5.5}$, Figures (3b) and (5b)

\section{Streamlines:}

Figures (6a)-(9b) show the graphs of streamlines for different values of time $\tau=0.5,1.0 \ldots 3.0$ for varying values of $\mathrm{Q}, \mathrm{C}, \mathrm{Pm}$ and $\mathrm{Pr}$ for both synchronous and asynchronous modulations. Furthermore it is observed that the influence of all the parameters for both the modulations on the contours size occurs only after some initial time

$\tau$. We also discern an important characteristic that the magnitude of stream function decreases from hot wall to cold wall.

In figures (6a) and (8a) we have presented the graphs for streamlines for varying values of time $\tau$ by varying values of $C$ and keeping the other parameters like Q, Pm, Pr and $\omega$ constant. In these figures for all values of $\tau$ and $\mathrm{C}$ it is observed that the streamlines are elliptic closed curves which lead to an interpretation that convection is in progress. It is also analyzed that the heat transfer due to convection hastens with increasing value of $\mathrm{C}$. Furthermore an increasing time $\tau$ decreases the stream function. Thus the parameter $\mathrm{C}$ increases the heat transfer. A similar behavior is observed for other parameters with the indication of progress of heat transfer in the system.

\section{CONCLUSIONS}

The effect of modulation in temperature on a weakly nonlinear Rayleigh- Bénard Chandrasekhar convection has been examined by employing the non-autonomous GinzburgLandau equation. The subsequent conclusions are extracted from the study:

1. There is a negligible effect on the amount of heat transfer in the system for synchronous modulation.

2. In the demonstration of synchronous modulation, there is a steady increase in $\mathrm{Nu}$ for small-scale values of time $\tau$; however for large values of $\tau$, the amount of heat transfer becomes constant.

3. Both Pr and Pm have an increasing effect with $\mathrm{Nu}$, thus for both the modulations there is an increase in the transfer of heat.

4. The Nusselt number shows an oscillatory behavior, in the demonstration of asynchronous modulation.

5. Initially for small scale values of $\tau$, the value of Nusselt number starts with 1, representing to the conduction state. Nevertheless with increasing $\tau, \mathrm{Nu}$ also increases, thus augmenting the heat transfer.

6. For all values of $\tau$ and the parameters C, Q, Pm and Pr for both synchronous and asynchronous modulation the streamlines are concentric closed curves.

7. Magnetic field is found to bring in a stability in the system.

8. Decrease in the values of stream function from the hot enclosure to the colder enclosure indicates the progress of convection and thus the transfer of heat in the system.

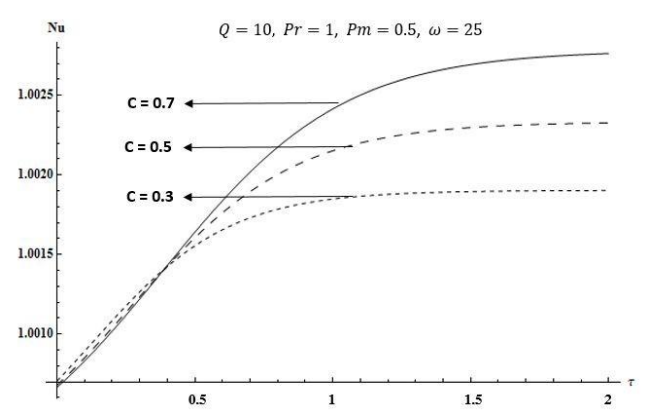

(b) Varying values of Couple Stress parameter, (C)

(a) Varying values of Chandrasekhar number, (Q)

Figure 2: Plot of time $(\tau)$ Vs Nusselt number $(\mathrm{Nu})$ for synchronous modulation 
International Journal of Applied Engineering Research ISSN 0973-4562 Volume 14, Number 2 (2019) pp. 523-533

(C) Research India Publications. https://dx.doi.org/10.37622/IJAER/14.2.2019.523-533

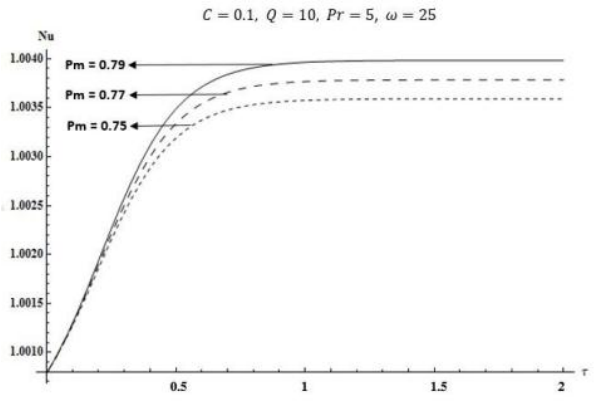

(a) Varying values of magnetic Prandtl number $(\mathrm{Pm})$

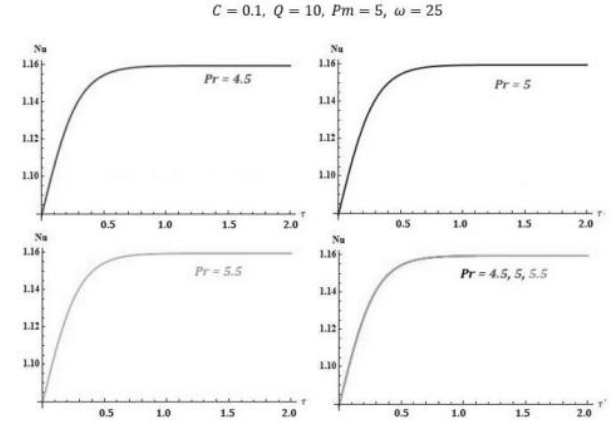

(b) Varying values of Prandtl number,

$(\operatorname{Pr})$

Figure 3: Plot of time $(\tau)$ Vs Nusselt number $(\mathrm{Nu})$ for synchronous modulation

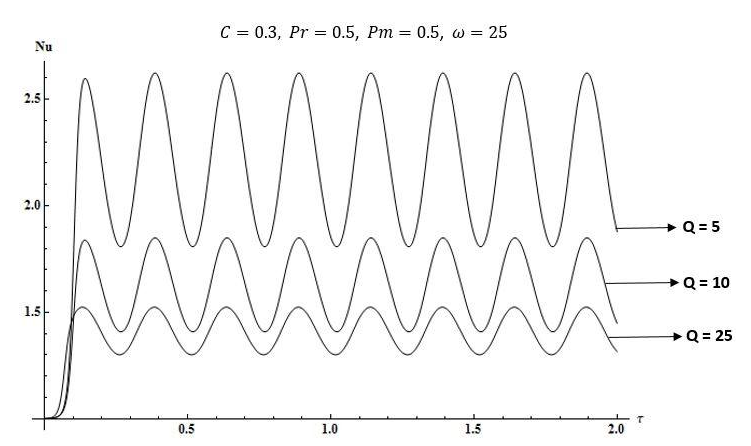

(a) Varying values of Chandrasekhar number (Q)

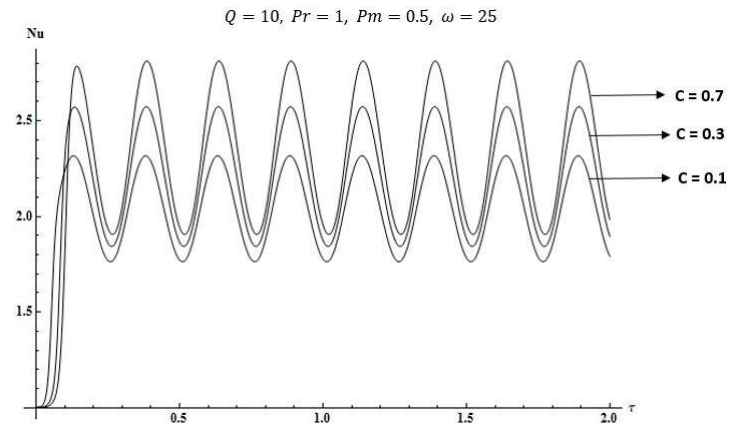

(b) Varying values of Parameter, $(\mathrm{C})$

Figure 4: Plot of time $(\tau)$ Vs Nusselt number $(\mathrm{Nu})$ for asynchronous modulation

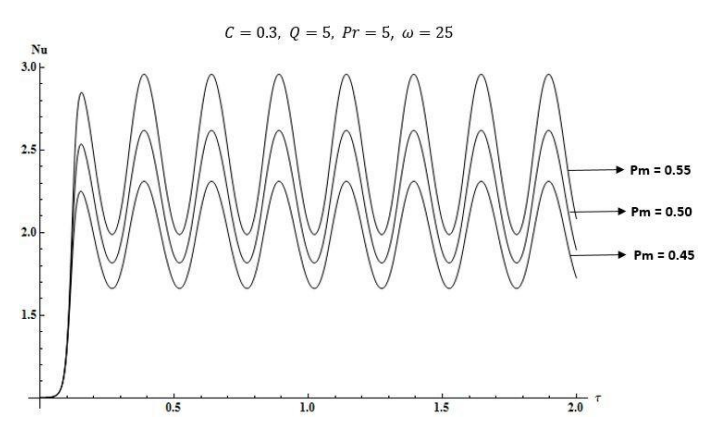

(a) Varying values of magnetic Prandtl number, $(\mathrm{Pm})$

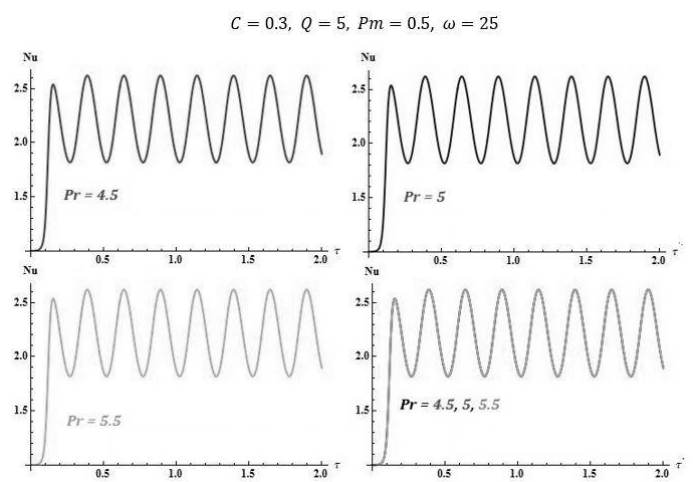

(b) Varying values of Prandtl number,

$(\operatorname{Pr})$

Figure 5: Plot of time $(\tau)$ Vs Nusselt number $(\mathrm{Nu})$ for asynchronous modulation 


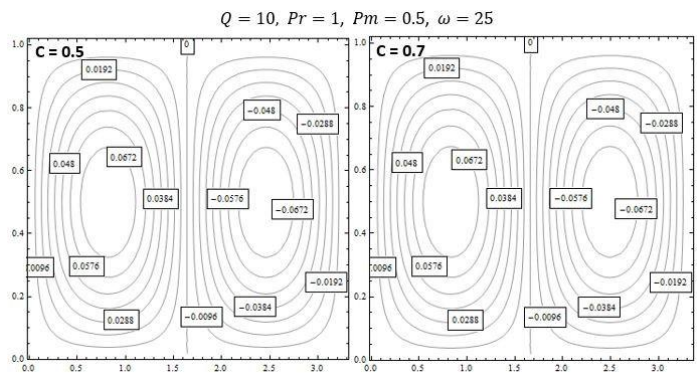

(a)Varying values of Couple Stress parameter, (C)

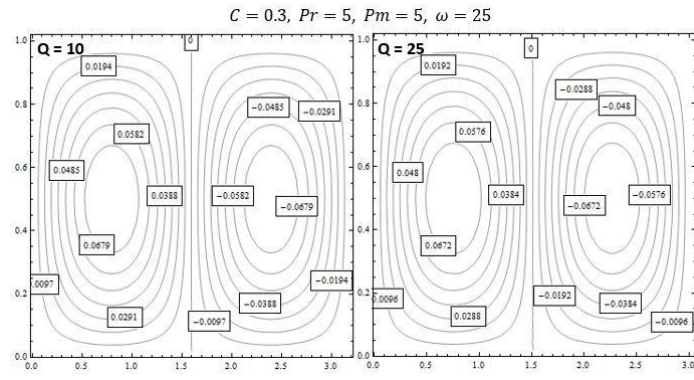

(b) Varying values of Chandrasekhar number, $(\mathrm{Q})$

Figure 6: Streamlines for synchronous modulation

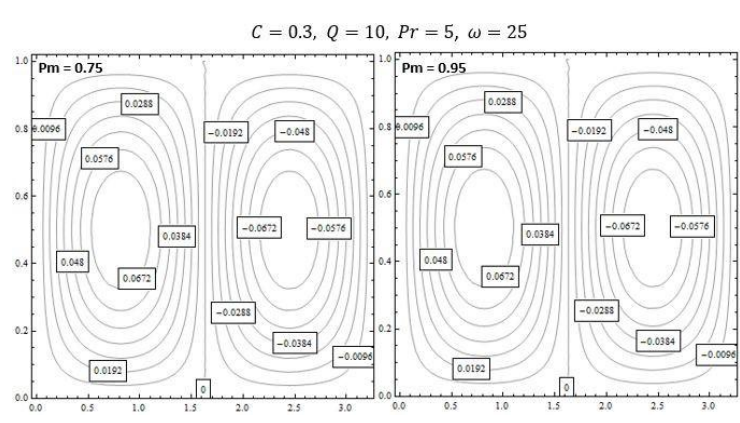

(a) Varying values of magnetic Prandtl number $(\mathrm{Pm})$

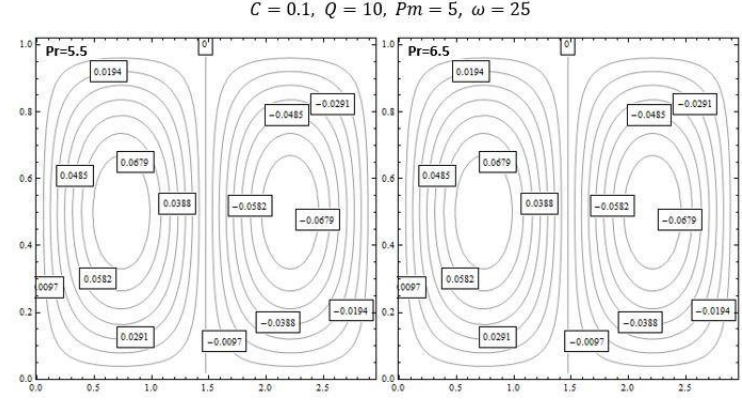

(b) Varying values of Prandtl number,

(Pr)

Figure 7: Streamlines for synchronous modulation

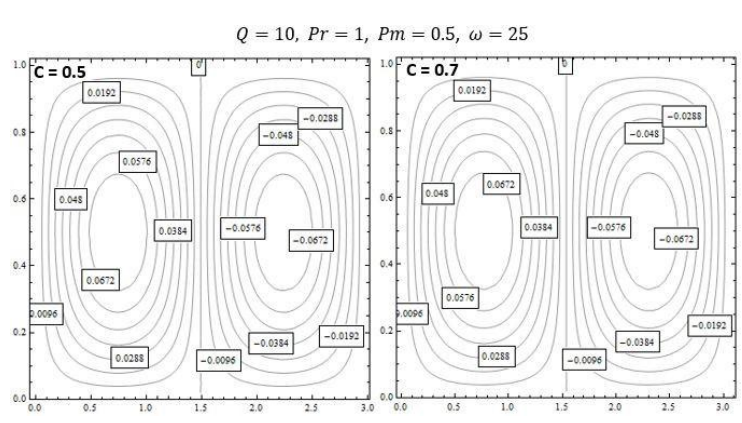

(a) Varying values of Couple stress parameter, (C)

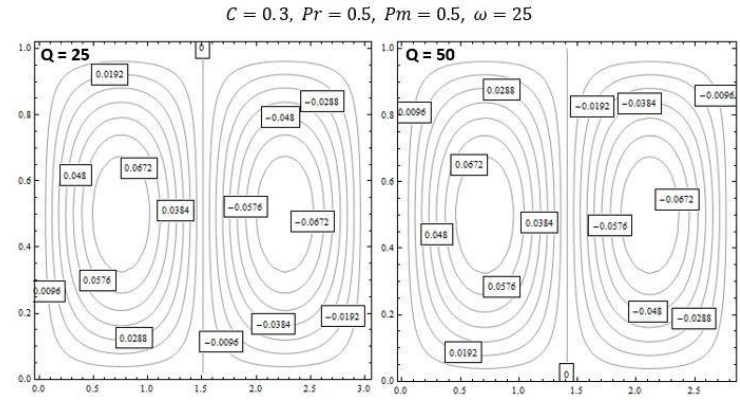

(b) Varying values of Chandrasekhar number, (Q)

Figure 8: Streamlines for asynchronous modulation

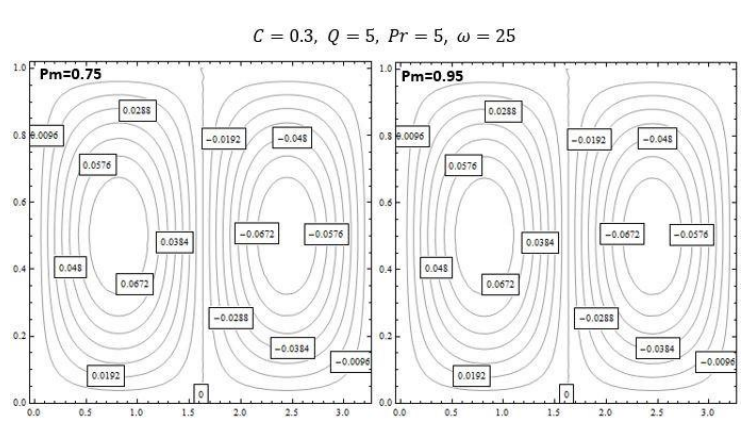

(a) Varying values of magnetic Prandtl number, (Pm)

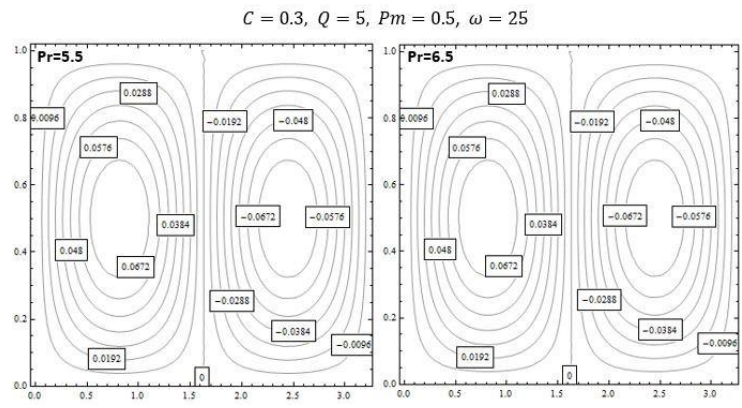

(b) Varying values of Prandtl number,

(Pr)

Figure 9: Streamlines for asynchronous modulation 
International Journal of Applied Engineering Research ISSN 0973-4562 Volume 14, Number 2 (2019) pp. 523-533

(C) Research India Publications. https://dx.doi.org/10.37622/IJAER/14.2.2019.523-533

\section{NOMENCLATURE}

a Dimensionless wave number

A Amplitude of convection

C Couple stress parameter

d Depth of the fluid

$\Delta \boldsymbol{T}$ Temperature difference between lower and upper $\operatorname{surfaces}\left(T_{1}-T_{0}\right)$

g Acceleration due to gravity $(0,0,-\mathrm{g})$

$H_{0} \quad$ Uniform magnetic field

$\vec{H} \quad$ Magnetic field

$H_{a} \quad$ Hartmann number

$\hat{\imath}, \hat{\jmath}, \widehat{k}, \quad$ Unit vectors in the $\mathrm{x}, \mathrm{y}, \mathrm{z}$ directions respectively

$H_{x}, H_{y}$ : Tangential components of magnetic field along $\mathrm{x}$ and $\mathrm{y}$ directions respectively

\section{$\vec{J} \quad$ Current density}

$1, \mathrm{~m}$ Horizontal components of wave number $\mathrm{Nu}$ : Nusselt number $\mathrm{p}$ : Pressure

P Magnetic pressure, $P=p+\frac{1}{2} \mu H_{0}^{2}$

Pm Magnetic Prandtl number

Pr Prandtl number

$\vec{q} \quad$ Velocity $(\mathrm{u}, \mathrm{v}, \mathrm{w})$

Q Chandrasekhar number

R Thermal Rayleigh number

$R_{0 c}$ Critical Rayleigh number $\mathrm{t}$ : Time

T Temperature

$T_{0} \quad$ Reference temperature

$T_{s} \quad$ Steady temperature Field

$(\mathrm{x}, \mathrm{y}, \mathrm{z}) \quad$ Cartesian coordinates with $\mathrm{z}$-axis vertically upwards $(\hat{\imath}, \hat{\jmath}, \widehat{k}$,$) : Unit vectors in the \mathrm{x}, \mathrm{y}$ and $\mathrm{z}$ directions respectively.

\section{Greek Symbols}

$\alpha$ Coefficient of thermal expansion

$\chi \quad$ Thermal conductivity

$\delta \quad$ Amplitude of modulation

$\delta^{2}$ Horizontal wave number,

$\delta_{1} \quad$ Perturbation parameter, $\delta_{1}^{2}=k_{c}^{2}+\pi^{2}$

$\gamma \quad$ Kinematic viscosity, $\gamma=\frac{\mu}{\rho_{0}}$

$\gamma_{m}$ Coefficient of magnetic viscosity, $\gamma_{m}=\frac{1}{\mu_{m} \sigma}$

$\mu$ Dynamic viscosity/coefficient of viscosity

$\mu$ Couple stress viscosity

$\mu_{m} \quad$ Magnetic permeability $\varphi \quad$ Phase angle

$\varphi_{H}$ Magnetic potential

$\varphi_{H^{*}}$ Dimensionless magnetic potential

$\rho \quad$ Fluid density

$\rho_{0} \quad$ Fluid density at the reference temperature $T_{0}$

$\psi \quad$ Stream function

$\psi^{*}$ Dimensionless stream function

$\tau \quad$ Slow time, $\tau=\epsilon^{2} t$

$\omega$ Frequency of modulation

$\epsilon^{2} \delta$ Small amplitude of modulation.

\section{Operators}

$\nabla \quad$ Vector differential operator $\left(\frac{\partial}{\partial x} \hat{i}+\frac{\partial}{\partial y} \hat{j}+\frac{\partial}{\partial z} \hat{k}\right)$

$\frac{\partial}{\partial t}$ Local time derivative

$\frac{D}{D t}$ Material or substantial derivative

$\nabla^{2}$ Three dimensional Laplacian operator

$\left(\frac{\partial^{2}}{\partial x^{2}}+\frac{\partial^{2}}{\partial y^{2}}+\frac{\partial^{2}}{\partial z^{2}}\right)$

$\nabla_{1}^{2}$ Two dimensional Laplacian operator $\left(\frac{\partial^{2}}{\partial x^{2}}+\frac{\partial^{2}}{\partial z^{2}}\right)$

\section{Subscripts and Superscripts}

b Basic state

c Critical value

$0 \quad$ Reference value

, Perturbed quantity

* Non-dimensional quantity

\section{REFERENCES}

[1] P. G. Drazin and W. H. Reid, Hydrodynamic Stability, 2nd ed. New York: Cambridge University Press, 1981.

[2] S. Chandrasekhar, Hydrodynamic and Hydromagnetic Stability, 3rd ed. New York: Oxford University Press, 1961.

[3] E. L. Koschmieder, Bénard cells and Taylor Vortices, New York: Cambridge University Press, 1993.

[4] E. Bodenschatz, W. Pesch and G. Ahler, Recent developements in Rayleigh-Bénard convection, Annu. Rev. Fluid Mech., vol. 32, pp. 709-778, 2000.

[5] D. A. Nield and A. Bejan, Convection in porous media, 3rd ed. New York:SpringerVerlag, 2006. 
[6] P.G. Siddheshwar, B. S. Bhadauria and O. P. Suthar, Synchronous and asynchronous boundary temperature modulations of Bénard Darcy convection, J. Magn. Mater., vol. 49, pp. 84-89, 2013.

[7] S. Pranesh, The effect Of imposed time-periodic boundary temperature and electric field on The onset of Rayleigh-Bénard convection in a micropolar fluid, Int. J. of Eng. Res. and Technol., vol. 2, no. 7, pp. 734-754, 2013.

[8] D. B.Ingham and I. Pop, Transport Phenomena in Porous Media III, 1st ed. Oxford:Elsevier, 2005.

[9] K. Vafai, Handbook of Porous Media, 3rd ed., New York:CRC Press, 2015.

[10] B.S. Bhadauria, P. Kiran, Weak nonlinear doublediffusive magneto-convection in a Newtonian liquid under temperature modulation, Int. J. Eng. Math., pp. 0114,2014

[11] S. Pranesh and G. Sangeetha, Effect of magnetic field on the onset of Rayleigh- Bénard convection in Boussinesq-Stokes suspensions with time periodic boundary temperatures, Int. J. of Appl. Math and Mech., vol. 6, no. 16, pp. 39-54, 2010.

[12] R. J. Donnelly, Experiments on the stability of viscous flow between rotating cylinders III. Enhancement of hydrodynamic stability by modulation, Proc. Roy. Soc., ser. A, vol. 281, pp. 130139, 1964.

[13] Venezian, Effect of modulation on the onset of thermal convection, J. Fluid Mech., vol. 35, pp. 243254, 1969.

[14] P. G. Siddheshwar and S. Pranesh, An analytical study of linear and non-linear convection in Boussinesq-Stokes suspensions, Int. J. Non-linear Mech., vol. 39, pp. 165$172,2004$.

[15] M. Lahmar and B. Bou-Sad, Couple stress effects on the dynamic behavior of connecting rod bearings in both gasoline and diesel engines, Tribol. Trans., vol. 51, pp. 44-56, 2008.

[16] N. B. Naduvinamani and S. B. Patil, Numerical solution of finite modified Reynolds equation for couple stress squeeze film lubrication of porous journal bearings, Comput. Struct., vol. 87, pp. 1287-1295, 2009.

[17] J. R. Lin and C. R. Hung, Combined effects of nonNewtonian couple stresses and fluid inertia on the squeeze film characteristics between a long cylinder and an infinite plate, Fluid Dyn. Res., vol. 39, pp. 616-631, 2007.

[18] E. F. El Shehawey and K. S. Mekhemer, Couple-stresses in peristaltic transport of fluids, J. Phys. Dyn. and Appl. Phys., vol. 27, pp. 1163 - 1165, 1994.

[19] A. C. Newell, and J. A. Whitehead, Finite bandwidth, finite amplitude convection, J. Fluid Mech., vol.28, pp. 279303, 1969.
[20] L. D. Landau and V. L. Ginzburg, On the theory of superconductivity, In Collected Papers of Landau,Pergamon Press. paper no.73, 1965.

[21] S. Govender, Stability of convection in a gravity modulated porous layer heated from below, Transport in Porous Media, vol. 57, no. 1, pp. 113123, 2004.

[22] P.G. Siddheshwar, B.S. Bhadauria P. Mishra and A.K. Srivastava, Study of heat transport by stationary magneto-convection in a Newtonian liquid under temperature or gravity modulation using GinzburgLandau model, Int. J. Non Linear Mech., vol. 47, pp. 418425, 2012.

[23] P.G. Siddheshwar, B.S. Bhadauria and A.K. Srivastava, An analytical study of nonlinear double diffusive convection in a porous medium under temperature/gravity modulation, Transport in Porous Media, vol. 91, pp. 585-604, 2012.

[24] W.V.R. Malkus, G. Veronis, Finite amplitude cellular convection, J Fluid Mech., vol. 4, 1958.

[25] P.G. Siddheshwar, B. S. Bhadauria and O. P. Suthar, Synchronous and asynchronous boundary temperature modulations of Bénard Darcy convection, J. Magn. Mater., vol. 49, pp. 84-89, 2013.

[26] S. Kaddeche, D. Henry and H. Benhadid, Magnetic stabilization of the buoyant convection between infinite horizontal walls with a horizontal temperature gradient, J Fluid Mech., vol. 480, pp. 185-216, 2003.

[27] A.A. Neha, Synchronous and Asynchronous Temperature Modulation Of Rayleigh- Bénard Chandrasekhar Convection in Couple Stress Fluid using Ginzburg-Landau Model, M.Phil Dissertation, Submitted to Christ University, May 2017. 